\title{
Towards Practical Applications of EQCN Experiments to Study Pt Anchor Sites on Carbon Surfaces
}

\author{
Adrian C. Fortuin ${ }^{1} \cdot$ Colleen Jackson $^{1} \cdot$ Emanuela Carleschi $^{2} \cdot$ Bryan P. Doyle $^{2}$. \\ Adam Shnier $^{3}$ - Roelof J. Kriek ${ }^{4}$ - Sekhar C. Ray ${ }^{5}$ - Dave G. Billing ${ }^{3}$. \\ Daniel Wamwangi $^{6}$ - Günther G. Scherer ${ }^{7}$ - Pieter B.J. Levecque ${ }^{1}$
}

Published online: 25 October 2017

(C) The Author(s) 2017. This article is an open access publication

\begin{abstract}
This work investigates the viability and outlines the current challenges in electrochemical quartz crystal nanobalance (EQCN) experiments on supported Pt catalysts. EQCN experiments involving Pt supported on 2-D "surface-treated graphite sputtered onto quartz crystal" (Pt/MFG-H) catalysts were compared to standard polycrystalline $\mathrm{Pt}\left(\mathrm{Pt}_{\text {poly }}\right)$, which showed similarities in frequency versus potential trends; however, the Pt/MFG-H catalysts obtained higher frequencies due to the support capacitance. The physical characterizations (XRD and
\end{abstract}

Student poster presentation at the ECAT2016 Symposium

Electronic supplementary material The online version of this article (https://doi.org/10.1007/s12678-017-0430-6) contains supplementary material, which is available to authorized users.

Pieter B.J. Levecque

pieter.levecque@uct.ac.za

1 HySA/Catalysis Centre of Competence, Catalysis Institute, Department of Chemical Engineering, University of Cape Town, Private Bag X3, Rondebosch 7700, South Africa

2 Department of Physics, University of Johannesburg, P.O. Box 524, Auckland Park, Johannesburg 2006, South Africa

3 School of Chemistry, University of the Witwatersrand, Johannesburg 2050, South Africa

4 Electrochemistry for Energy and Environment Group, Research Focus Area: Chemical Resource Beneficiation (CRB), North-West University, Potchefstroom 2531, South Africa

5 Department of Physics, College of Science, Engineering and Technology, University of South Africa, Private Bag X6, Florida 1710, South Africa

6 School of Physics, University of the Witwatersrand, Johannesburg 2050, South Africa

75607 Hägglingen, Switzerland
XPS) and electrochemical responses, mainly cyclic voltammetry in acidic media and the ferri/ferrocyanide couple, of the 2-D Pt/MFG-H were compared to the representative 2-D Pt supported on treated highly orientated pyrolytic graphite (Pt/HOPG-H), in order to make assertions on the similarities between the two catalysts. The XRD diffraction patterns and the XPS valence band structure for the treated and untreated MFG (-H and -P, respectively) and HOPG (-H and $-\mathrm{P}$, respectively) demonstrated similarities. Nevertheless, the cyclic voltammograms and peak positions of the ferri/ferrocyanide couple between the treated and untreated MFG and HOPG catalysts were dissimilar. However, EQCN may be used qualitatively between the two different 2-D catalysts since the same trends in electrochemical responses before and after treatment of the MFG and HOPG catalysts were seen. Hence, the EQCN technique can be used in future studies as an alternative method to study degradation mechanisms of $\mathrm{Pt}$ and carbon for PEFCs.

Keywords EQCN · Graphite · Cyclic voltammetry · XPS · $\mathrm{XRD} \cdot$ Carbon electrochemistry

\section{Introduction}

Electrochemical quartz crystal nanobalance (EQCN) is a powerful tool to study interfacial electrochemistry phenomena [1,2]. This technique allows for conventional electrochemical techniques, such as cyclic voltammetry (CV), chronoamperometry (CA), and other potential sweep methods to be used in parallel with the detection of nanogram mass changes [3-5]. This leads 
to detail study of electrochemical surface reaction mechanisms, deposition rates, and roughness effects [5-7]. The technique has been shown to be particularly useful in sensor applications, as its sensitivity allows for small mass/concentration changes in a system to be easily detected [8-10].

Much of the research in the electrocatalysis field has not been able to make use of this powerful tool as real-world catalysts cannot easily be applied to a quartz crystal resonator essential for EQCN experiments. Fundamental research, such as the work conducted by Jerkiewicz et al. [11-13], has focused on using the EQCN technique to model a carbon supported platinum $(\mathrm{Pt} / \mathrm{C})$ electrocatalyst as a bulk polycrystalline $\mathrm{Pt}\left(\mathrm{Pt}_{\text {poly }}\right)$ catalyst. While this gives critical insights into the surface reaction mechanisms on Pt, it excludes the metalsupported interactions between Pt and carbon. However, little work has been published with regard to modifying a quartz crystal resonator for more applied investigations into the interfacial electrochemistry occurring on supported platinum nanoparticles and the electronic interaction between $\mathrm{Pt}$ and carbon in a Pt/C electrocatalyst.

Some attempts have been made at depositing a Pt/C ink onto a gold-coated quartz crystal [14, 15]. However, these systems are difficult to calibrate since precise loadings are required to ensure that the quartz crystal oscillations are not drastically different from measurement to measurement.

Before directly studying Pt anchor sites on carbon by EQCN, we first need to consolidate the above by showing that we can directly compare two different 2-D model Pt/C catalyst systems, such that the stability (and hence anchoring) of Pt on the support can be investigated by EQCN. The model system of the realworld catalyst (e.g., Pt supported on Vulcan) is represented by Pt sputtered onto basal plane oxygen terminated highly orientated pyrolytic graphite (HOPG). The system of comparison is a mirror-finished graphite (MFG) quartz crystal resonator, onto which Pt has been sputtered. The MFG undergoes the exact same surface oxidation treatment as the HOPG, such that the two catalysts can be directly compared. The aim is to obtain similar electrochemical responses on the MFG as HOPG, using conventional electrochemical methods, such that we can include EQCN measurements in future studies. This report studies the physical characteristics of the two systems, namely, the crystallite size, crystallography, surface functional groups, and electrochemical responses via cyclic voltammetry in acidic media and the ferri/ferrocyanide couple $[16,17]$.

\section{Experimental}

All electrochemical measurements were conducted using a Bio-Logic SP-200 potentiostat. EQCN measurements were conducted using a Seiko EG\&G QCM922A, in a home-built Faraday cage and vibration free table. The packaged EC-Lab v11.10 software was used to control both instruments.

\section{Electrodes}

A custom-built, two-compartment cell was used and cleaned by submerging in NOCHROMIX® (Godax Laboratories, Inc.) overnight. A Teflon ${ }^{\mathrm{TM}}$ quartz holder houses the planar quartz crystals cut in the AT orientation, in the vertical cell orientation as described by Jerkiewicz et al. [18]. An AT-cut quartz crystal was coated with a 100-nm Ti layer, followed by sputtering of approximately 300-nm Pt layer to form a Pt-coated quartz crystal (PQC). Alternatively, a 300-nm carbon layer was sputtered onto the 100-nm Ti layer on the quartz to fabricate a MFG quartz crystal with a roughness of $60 \mathrm{~nm}$. The base resonant frequency was 9.00 and $9.13 \mathrm{MHz}$ for $\mathrm{PQC}$ and MFG, respectively. All quartz crystals were supplied by Bio-Logic.

The working electrode for the conventional system consisted of $1 \mathrm{~cm}^{2}, 200-\mu \mathrm{m}$-thick HOPG disks (Optigraph $\mathrm{GmbH}$, Berlin). This was attached to a conventional glassy carbon (GC) electrode using a carbon-Nafion ${ }^{\circledR}$ (Ion Power) paste. The HOPG surface was modified by refluxing in Fenton's reagent for $24 \mathrm{~h}$ at $80{ }^{\circ} \mathrm{C}$ [19]. The hydroxylmodified HOPG is referred to as HOPG-H, with the pristine HOPG samples referred to as HOPG-P. Pt was then deposited onto the modified and unmodified surfaces at a loading of $20 \mu \mathrm{g} \mathrm{cm}^{-2}$ by DC magnetron sputtering (PVD Products, USA). This was accomplished by using a Pt sputtering target (1.5" diameter $\times 1 / 8$ " thick) obtained from ACI Alloys (USA) having a purity of $99.95 \%$. Prior to sputtering, the vacuum chamber of the sputtering apparatus was pumped down to a base pressure of approximately $5 \times 10^{-7} \mathrm{Torr}$. Sputtering of Pt, under an Ar plasma, was conducted at a chamber pressure of $8 \mathrm{mTorr}$ and an Ar flow of $15 \mathrm{sccm}$. The sputtering rate $\left(\mathrm{nm} \mathrm{min}{ }^{-1}\right)$ of Pt was calibrated as described by Falch et al. [20]. The substrates were positioned onto a stainless steel tray that slots into housing fitted with a computer-controlled XY motor below a fixed aperture, allowing for dedicated sputtering of individual samples. Similarly, the aforementioned procedure was used to prepare surface-modified MFG and deposit Pt. The hydroxyl-modified MFG is referred to as MFG-H and the unmodified MFG is referred to as MFG-P. In this instance, a dedicated stainless steel tray was manufactured in-house to deposit Pt on the MFG samples in an evenly spaced manner within the sputtering apparatus.

A reversible hydrogen electrode (RHE) using Pt foil (Alfa Aesar, 99.99\%) was used as the reference electrode and a Pt mesh (Alfa Aesar, 99.99\%) as the counter electrode.

\section{Electrochemical Testing}

Prior to conducting electrochemical measurements on HOPG and MFG, cyclic voltammograms ( $i$ vs $E$ ) of polycrystalline $\mathrm{Pt}$ were measured in aqueous $0.5 \mathrm{M} \mathrm{H}_{2} \mathrm{SO}_{4}$ prepared from $99.99 \mathrm{wt} \% \mathrm{H}_{2} \mathrm{SO}_{4}$ (Sigma-Aldrich) and $18.2 \mathrm{M} \Omega \mathrm{cm}$ 
nanopure water. All measurements were carried out at room temperature and under an inert Ar atmosphere (99.99\%, Air Products). The electric potential was cycled between 0.05 and $1.50 \mathrm{~V}$ vs RHE to ensure cleanliness and reproducible functioning of the electrochemical setup.

To confirm the oxidation of HOPG by various treatment methods and changes in the electronic states of the carbon, $\mathrm{CVs}$ using the ferri/ferrocyanide redox couple were run. A solution of $1 \mathrm{mM} \mathrm{K}_{4} \mathrm{Fe}(\mathrm{CN})_{6}$ (Sigma-Aldrich) in $1 \mathrm{M} \mathrm{KCl}$ (Merck) was prepared. The electric potential was cycled between 0.00 and $1.20 \mathrm{~V}$ vs RHE at scan rates of 100, 75, 50, 25, and $20 \mathrm{mV} \mathrm{s}^{-1}$, of which we only report on the scan rate at $50 \mathrm{mV} \mathrm{s}^{-1}$ here. See Fig. S1 and S2 for current responses for all scan rates and summaries of $\Delta E_{p}$ and peak current density $\left(j_{p}\right)$ as a function of scan rates. Similarly, this was applied to the MFG system and the two systems were compared. Because this involves an inner-sphere electron transfer reaction $[16,17]$ at the electrode surface, frequency vs potential data were not collected.

\section{Physical Characterization}

X-ray diffraction (XRD) measurements were taken using a Bruker D2 Phaser in the Bragg-Brentano geometry with a Co anode $(\lambda=1.79026 \AA)$, primarily using a $2 \theta$ range of $20-90^{\circ}$ with $0.0274^{\circ}$ steps. Samples were placed in a zero background sample holder and the sample was placed such that they were in line with a straight edge across the holder. The measurement was repeated at various angles $(\Phi)$ about the vector normal to the sample surface.

Room temperature X-ray photoelectron spectroscopy (XPS) measurements were carried out using a monochromated Al source $(1487.1 \mathrm{eV})$ and a SPECS PHOIBOS 150 hemispherical electron energy analyzer. The overall experimental resolution was approximately $0.6 \mathrm{eV}$ for all spectra. Some of the spectra were acquired on a KRATOS-SUPRA spectrometer at UNISA (Florida Science Campus, South Africa), using a monchromated $\mathrm{Al}$ source $(1486.6 \mathrm{eV})$ having a base pressure of $1.2 \times 10^{-8}$ Torr. The surface of all samples was cleaned with a short cycle of Ar ion sputtering. This ranged from 30 to $120 \mathrm{~s}$, with measurements taken at various intervals.

\section{Results and Discussion}

\section{Physical Characterization}

XRD measurements showed reproducible peak shapes across the lateral position of the various carbon substrates. The patterns were independent of the orientation about $\Phi$, by considering the reproducibility of the peak shape and intensities (e.g., HOPG-H (d)-(f) peaks in Fig. 1), which shows that the scattering vector was normal to the surface (Fig. 1).
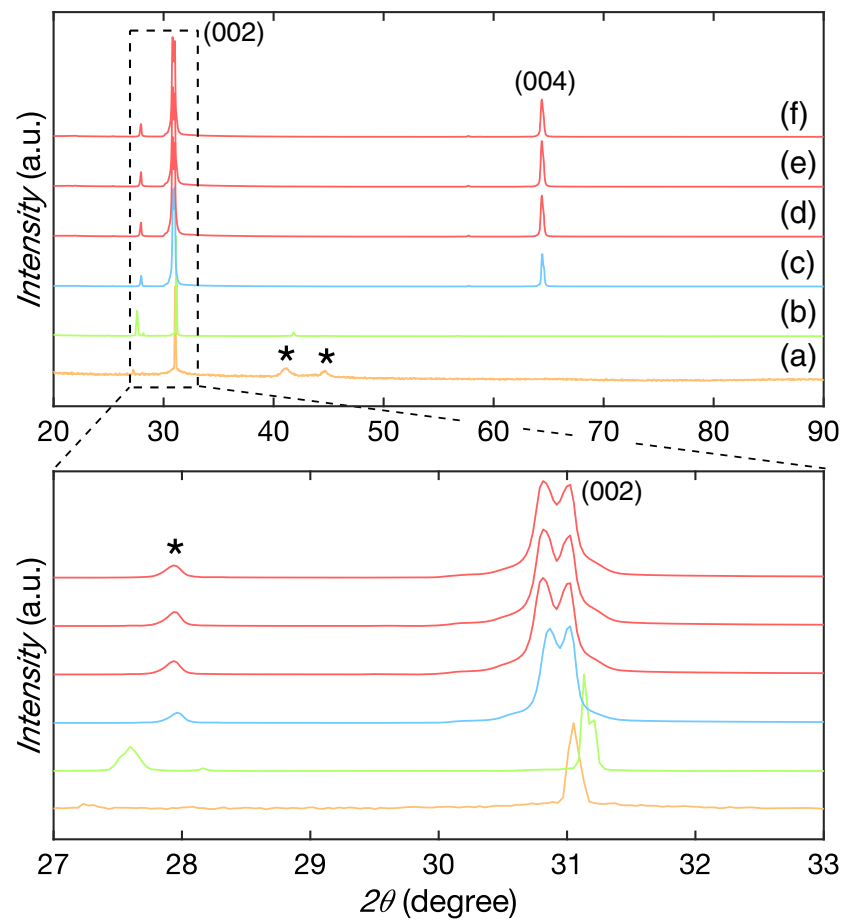

Fig. 1 Diffractograms comparing the various carbon substrates where (a) MFG-P, (b) MFG-H, (c) HOPG-P, and (d)-(f) HOPG-H at different angles of $\Phi$. The magnified portion focuses on the graphite (002) orientation, showing a shift to higher $d$-spacing for the MFG substrates

Furthermore, the MFG graphitic peaks around $31^{\circ} 2 \theta$ are shifted to a larger angle compared to the HOPG samples, suggesting a slightly smaller $\mathrm{d}$-spacing. This was accompanied by narrower peak widths relating to a larger crystallite size. Relative crystallite sizes were estimated using the Scherrer equation [21], as seen in Table 1. Absent peaks in the MFG samples suggest a preferred orientation, while this does not appear the case for the HOPG samples with which they were compared. Furthermore, the peaks indicated by $*$ suggest small quantities of nitrogen and oxygen within the carbon lattice.

XPS measurements confirm the results seen in XRD, with a sharp C $1 s$ peak observed in the survey spectra (Fig. 2). There was minimal nitrogen and oxygen present in the

Table 1 Comparison of graphite crystallite size and Pt crystallite size as determined by the Scherrer equation [21] of HOPG and MFG substrates at loadings of $50 \mu \mathrm{g} \mathrm{cm}^{-2} \mathrm{Pt}$

\begin{tabular}{lll}
\hline Substrate & $\begin{array}{l}\text { Graphite (002) } \\
\text { crystallite size }\end{array}$ & $\begin{array}{l}\text { Pt (111) } \\
\text { crystallite size }\end{array}$ \\
\hline HOPG-P & $26.1 \mathrm{~nm}$ & - \\
MFG-P & $63.0 \mathrm{~nm}$ & - \\
Pt/HOPG-P & $23.0 \mathrm{~nm}$ & $10.8 \mathrm{~nm}$ \\
Pt/HOPG-H & $26.7 \mathrm{~nm}$ & $10.1 \mathrm{~nm}$ \\
Pt/MFG-P & $71.3 \mathrm{~nm}$ & $14.9 \mathrm{~nm}$ \\
Pt/MFG-H & $85.9 \mathrm{~nm}$ & $12.1 \mathrm{~nm}$ \\
\hline
\end{tabular}




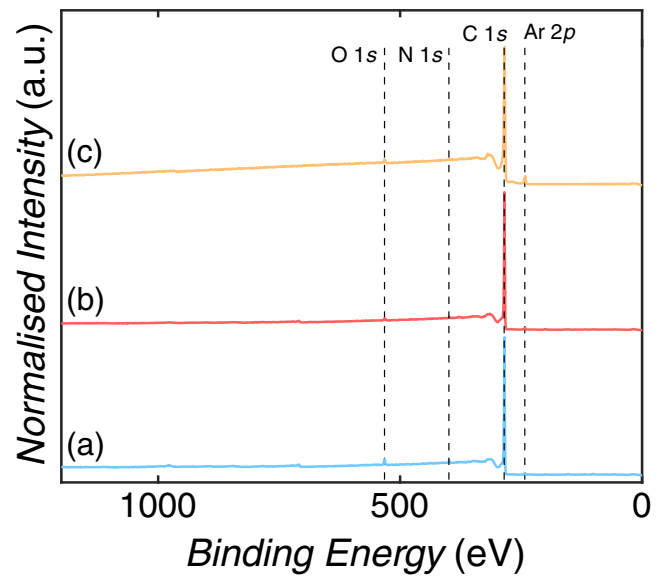

Fig. 2 XPS survey spectra of (a) HOPG-P, (b) HOPG-H, and (c) MFG-P. Bands at $532.4 \mathrm{eV}, 398.5 \mathrm{eV}, 284.4 \mathrm{eV}$, and $241.1 \mathrm{eV}$ are assigned to $\mathrm{O} 1 s$, $\mathrm{C} 1 s, \mathrm{~N} 1 s$, and $\mathrm{Ar} 2 p$, respectively

samples, with only an appreciable contaminant present in the MFG-P spectrum. This small Ar $2 p$ peak at approximately $241.1 \mathrm{eV}$ is due to Ar implantation in the graphite matrix during sputtering [22].

Based on the valence band data displayed in Fig. 3, all samples have similar densities of state (DOS) near the Fermi level $\left(E_{F}\right)$ as indicated by the overlay of the spectra in the inset. However, MFG-P does have a more pronounced O $2 s$ band at $21.9 \mathrm{eV}$, suggesting that this graphite moiety is covered with larger amounts of surface carbonyl groups as stated in other studies [23-25]. This observation is further supported by the broadening of MFG-P's C $1 s$ spectrum towards higher binding energies shown in Fig. 4.

\section{Electrochemical Results}

$\left[\mathrm{Fe}(\mathrm{CN})_{6}\right]^{4-/ 3-}$ Redox Couple for Carbon Substrates

The CVs of the ferri/ferrocyanide redox couple were studied for different graphite substrates, shown in Fig. 5. Control

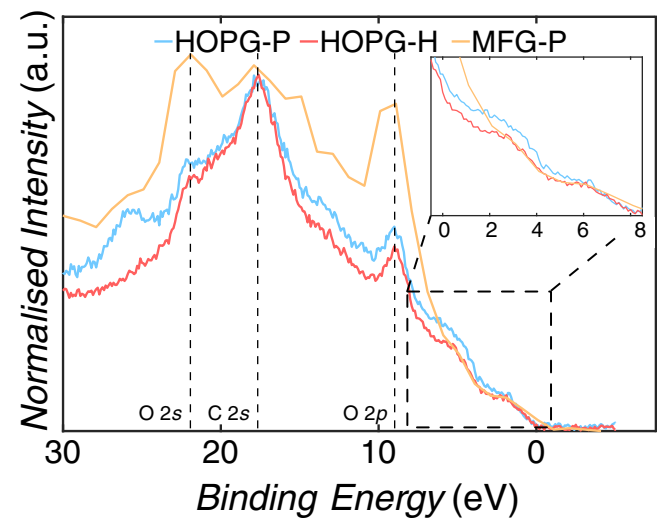

Fig. 3 Valence band spectra of HOPG-P, HOPG-H, and MFG-P, respectively. Bands at $22.2-21.9 \mathrm{eV}, 17.9-17.6 \mathrm{eV}$, and $9.0-8.9 \mathrm{eV}$ are assigned to $\mathrm{O} 2 s, \mathrm{C} 2 s$, and $\mathrm{O} 2 p$, respectively. The inset shows a magnification of the densities of state closer to the Fermi level in UHV

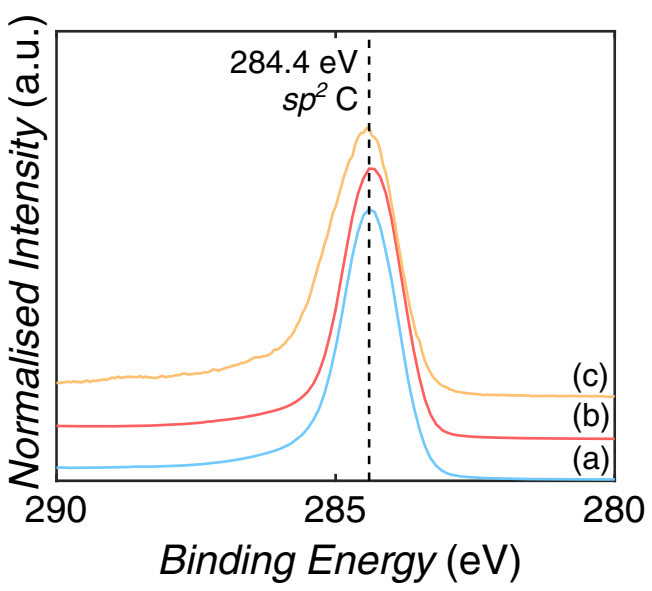

Fig. $4 \mathrm{C} 1 s$ spectra of (a) HOPG-P, (b) HOPG-H, and (c) MFG-P. All spectra are calibrated to $284.4 \mathrm{eV}$ as the $\mathrm{C} s p^{2}$ band

substrates of GC, HOPG-P, and MFG-P were studied to create a baseline to which surface modifications and Pt depositions can be compared. From the corresponding CVs, an estimated electrode capacitance $(C)$ [26] was determined, and the rate constant, $k^{0}$, using Nicholson's method [27].

Unmodified, pristine HOPG surfaces show slow rates of electron transfer for the $\left[\mathrm{Fe}(\mathrm{CN})_{6}\right]^{4-/ 3-}$ couple. This has been postulated by Cline et al. [17] to be due to a lack of surface reactive sites as the basal plane of HOPG, which is not a suitable electron transfer structure. With the introduction of surface disorder, i.e., by surface treatment/oxidation, there is a significant increase in the rate constant as indicated in Table $2[16$, 17]. Furthermore, as seen in Fig. $5 \mathrm{a}$, the broadening of the HOPG-P peaks ( $E_{p, a}$ and $E_{p, c}$ ) and deviation from the classic "duck bill" shape suggest that there are few sites for adsorption for the ferrocyanide ion and subsequent electron transfer.

The HOPG-H substrate shows a fourfold increase in capacitance over HOPG-P (Fig. 5a). This is a similar capacitance value observed as for GC. This increase is most likely due to decreased conductivity of HOPG-H. However, since the resolution of the $\mathrm{Al} \mathrm{X}$-ray source is too low to see such small changes in the near- $E_{F}$ electronic structure, it is speculated that the process of oxidizing HOPG exfoliates a few layers of graphite from the surface while the hydroxyl radicals bond to the surface. The fact that HOPG-P and HOPG-H show very similar XPS spectra after Ar ion sputtering is evidence that only the very top few atomic layers of HOPG participate in oxidation.

Contrary to HOPG, as shown in Fig. 5b, d, the large increase in capacitance is not seen for the MFG-H samples as seen between HOPG-P and HOPG-H. Although not well understood, it is postulated that the surface orientation of the graphite is slightly different from HOPG, as seen in $\mathrm{XRD}$, and thus less hydroxyl radicals react with the surface. What is clear, though, is that HOPG-H and MFG-H show increased electron transfer rates over their HOPG-P and MFG-P counterparts. 
Fig. 5 Current responses of (a) HOPG-P and HOPG-H, (b) MFG-P, MFG-H, and GC, (c) HOPG-P and MFG-P, and (d) HOPG-H and MFG-H in $1 \mathrm{mM}$ $\left[\mathrm{Fe}(\mathrm{CN})_{6}\right]^{4-} / 1 \mathrm{M} \mathrm{KCl}_{(\mathrm{aq})}$ solution under $\operatorname{Ar}_{(\mathrm{g})}$ saturation and room temperature, with a sweep rate of $50 \mathrm{mV} \mathrm{s}^{-1}$

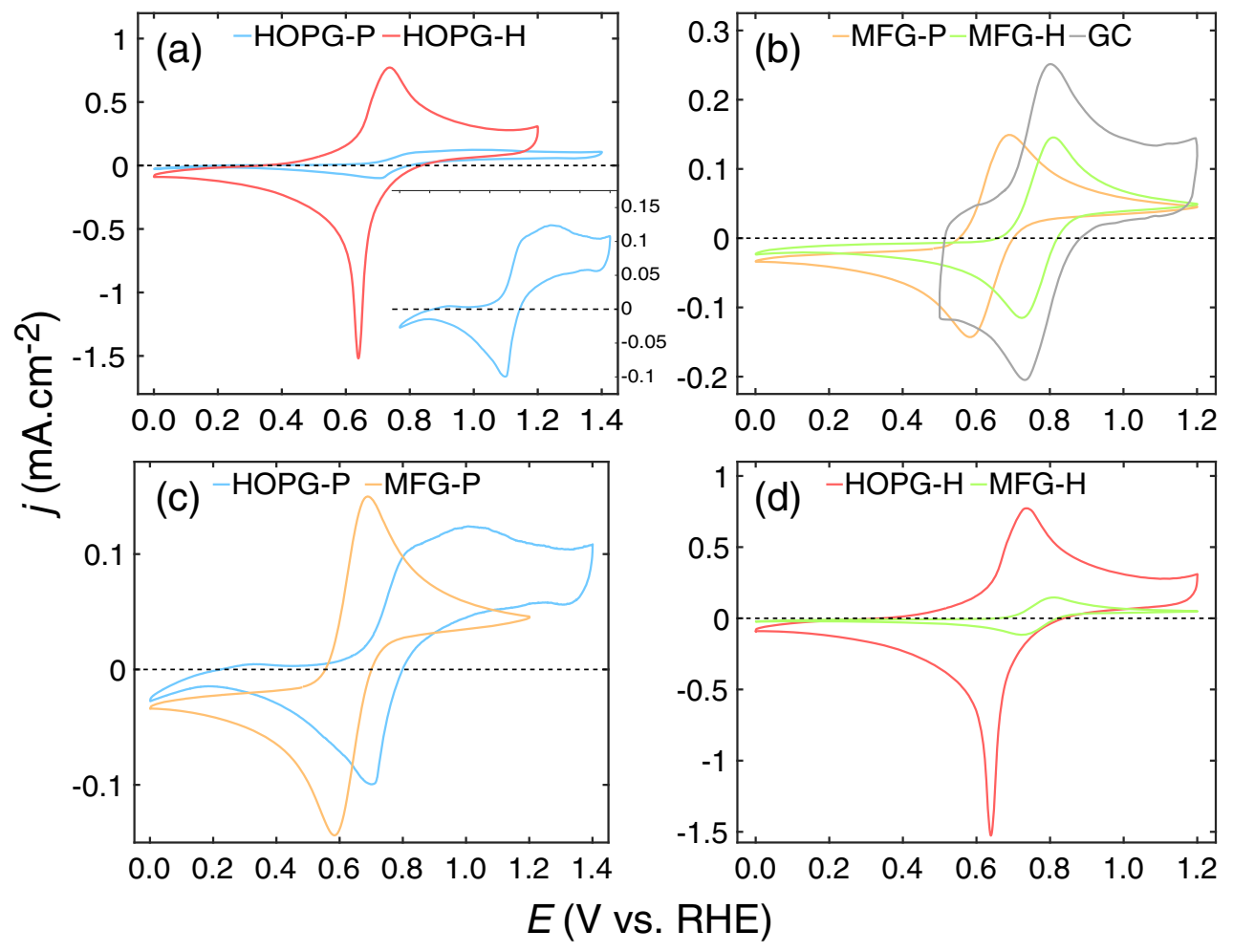

\section{Electrochemical Response of Pt-Supported Carbon Substrates}

A loading of $20 \mu \mathrm{g} \mathrm{cm}^{-2}$ Pt was deposited onto the various carbon substrates as described in the "Electrodes" section. CVs in an acidic medium were recorded for characteristic Pt electrochemical response and CVs in ferri/ferrocyanide were recorded and compared as in the "[Fe(CN)6]4-/3- Redox Couple for Carbon Substrates" section.

Table 2 Redox potential, kinetic data, and capacitance values for various carbon substrates with loadings of $20 \mu \mathrm{g} \mathrm{cm}^{-2} \mathrm{Pt}$ and without Pt (see supplementary note 1 for an explanation of how $k^{0}$ and $C$ were calculated)

\begin{tabular}{llll}
\hline Substrate & $\Delta E_{p}(\mathrm{mV})$ & $k^{0}\left(\mathrm{~cm} \mathrm{~s}^{-1}\right)^{\mathrm{a}}$ & $C\left(\mu \mathrm{F} \mathrm{cm}{ }^{-2}\right)$ \\
\hline HOPG-P & 306 & $5.0 \times 10^{-7}$ & 750 \\
HOPG-H & 95.2 & 0.0056 & 3200 \\
HOPG-P20 & 65.0 & 0.035 & 734 \\
HOPG-H20 & 75.0 & 0.014 & 1270 \\
MFG-P & 107 & 0.0033 & 230 \\
MFG-H & 85.9 & 0.0085 & 310 \\
MFG-P20 & 87.4 & 0.0080 & 127 \\
MFG-H20 & 185 & $1.1 \times 10^{-4}$ & 143 \\
GC & 68.7 & 0.020 & 3000 \\
\hline
\end{tabular}

${ }^{\mathrm{a}} \alpha=0.5, D_{0}=D_{R}=10^{-5} \mathrm{~cm}^{2} \mathrm{~s}^{-1}$
As expected with the deposition of Pt, the capacitance of the samples decreases. From Fig. 6a HOPG-P20 and MFGP20 (20 $\mu \mathrm{g} \mathrm{cm}^{-2} \mathrm{Pt}$ deposited), the Pt CVs indicate similar responses, with the only appreciable difference in the oxide formation region. The onset of oxide formation is shifted to higher potentials in MFG-P20, indicating that this graphite moiety may have larger Pt nanoparticles deposited and is more oxophilic [28]. However, Fig. 6b seems to suggest that the Pt layer may be shielded (i.e., implanted in the graphite matrix), as there is larger current contribution from graphite in the $\left[\mathrm{Fe}(\mathrm{CN})_{6}\right]^{4-/ 3-}$ redox couple. The greater surface roughness of MFG as compared to HOPG supports the above hypothesis and supports the reasoning that inner-sphere electron transfer is taking place, as opposed to outer sphere electron transfer. The ill-defined $\mathrm{Pt}_{\text {Hupd }}(0.0-0.4 \mathrm{~V}$ vs $\mathrm{RHE})$ region and larger $\Delta E_{p}$ (Table 2) is a clear indicator that Pt plays less of a role in electron transfer than what is suggested by the current response in acid. Similarly, for the peroxide-treated equivalent samples (Fig. 6c, d), the above trend is observed. Furthermore, the tilting of HOPG-H20's current response in acid (Fig. 6c) is indicative of decreased conductivity within the support.

\section{Frequency Response of Pt Supported on Carbon Substrates}

While the above has shown that Pt/MFG has a typical current response for $\mathrm{Pt}$ in acid media, its frequency response needs to be compared to a baseline of $\mathrm{Pt}_{\text {poly }}$-coated quartz crystal. The frequency response curve of $\mathrm{Pt}_{\text {poly }}$ has been well documented 
Fig. 6 Comparative current responses of $20 \mu \mathrm{g} \mathrm{cm}^{-2} \mathrm{Pt}$ on HOPG-P, MFG-P, HOPG-H, and MFG-H in (a), (c) $0.5 \mathrm{M} \mathrm{H}_{2} \mathrm{SO}_{4}$ and (b), (d) $1 \mathrm{mM}\left[\mathrm{Fe}(\mathrm{CN})_{6}\right]^{4}$ - $/ 1 \mathrm{M} \mathrm{KCl}_{(\mathrm{aq})}$ solution under $\operatorname{Ar}_{(\mathrm{g})}$ saturation and room temperature, with a sweep rate of $50 \mathrm{mV} \mathrm{s}^{-1}$

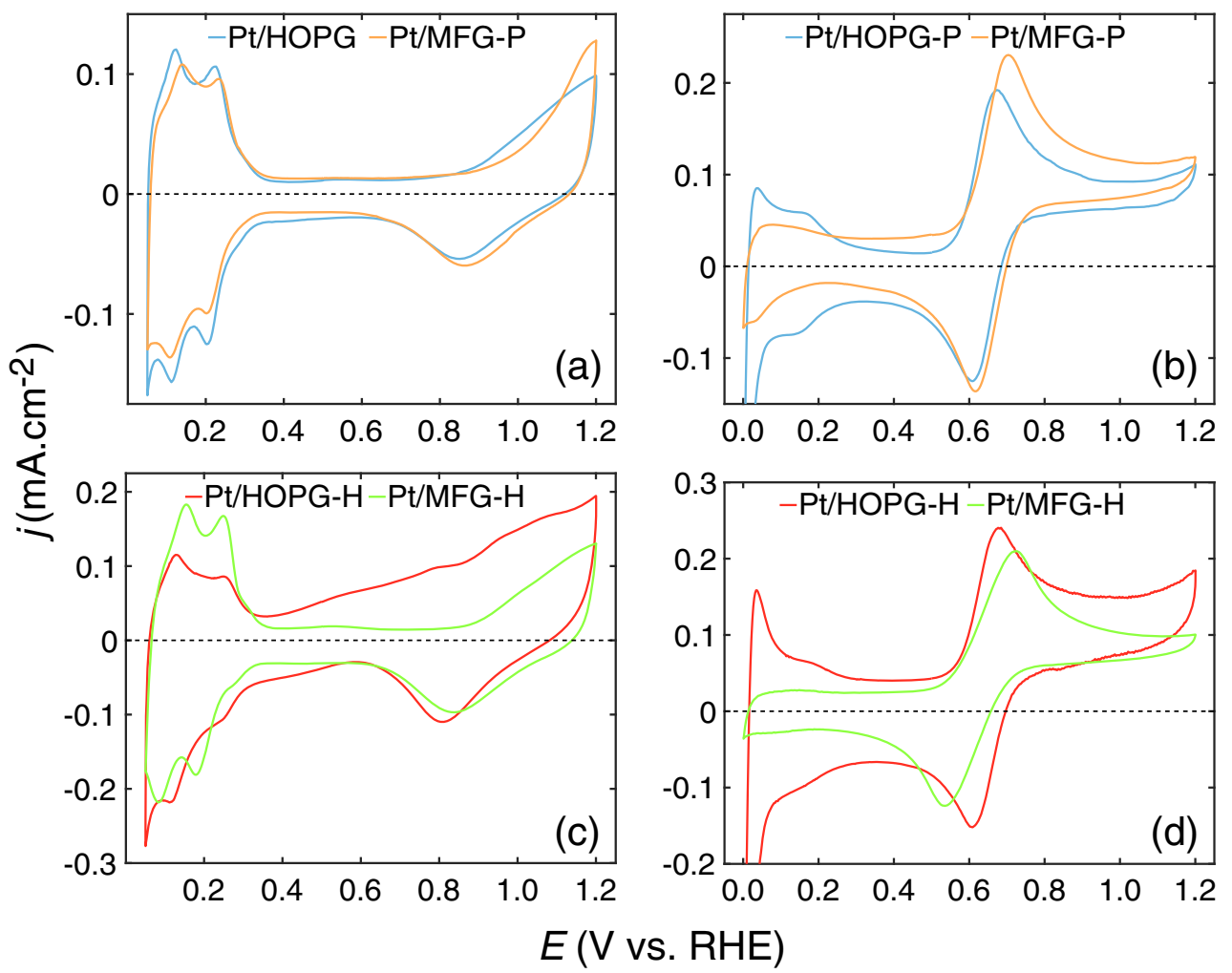

by Jerkiewicz et al. [4, 11-13, 18, 29, 30] over the years, and an accurate reproduction of the standard Pt frequency response serves as the basis of validating the EQCN setup.

From Fig. 7, a frequency response for $\mathrm{Pt}_{\text {poly }}$ over a range of $0.0-1.5 \mathrm{~V}$ vs RHE shows a frequency change of approximately $25 \mathrm{~Hz}$, which is in good accordance to that reported by Jerkiewicz et al. [12] and Kim et al. [30].

Measuring the frequency response of MFG-H20 against $\mathrm{Pt}_{\text {poly }}$, a clear similarity between the two catalysts can be seen, but a larger $\Delta f$ response versus potential in the graphite substrate is observed. This is to be expected, as the surface of MFG-H20 has a significantly larger capacitance than Pt, hence a larger effect of ion attraction and repulsion from the

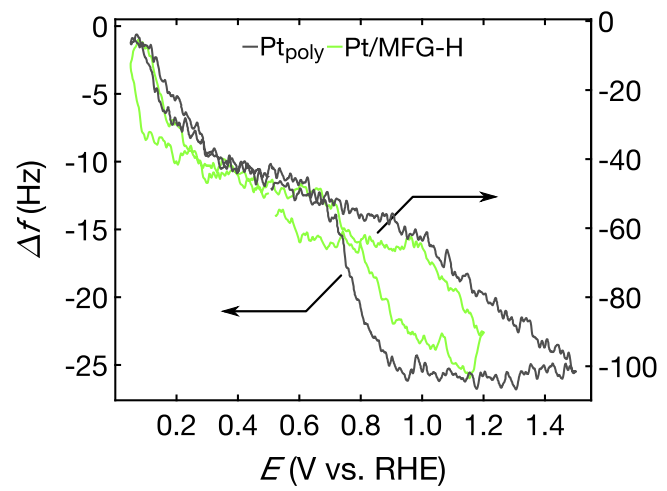

Fig. 7 Comparative frequency response of $\mathrm{Pt}_{\text {poly }}$ quartz crystal and $20 \mu \mathrm{g} \mathrm{cm}^{-2} \mathrm{Pt} / \mathrm{MFG}-\mathrm{H}$ quartz crystal in $0.5 \mathrm{M} \mathrm{H}_{2} \mathrm{SO}_{4}$ under $\mathrm{Ar}_{(\mathrm{g})}$ saturation and room temperature, with a sweep rate of $50 \mathrm{mV} \mathrm{s}^{-1}$ surface influencing the response as discussed by Jerkiewicz [12]. This is further substantiated by the wider hysteresis in the $\mathrm{Pt}_{\mathrm{Hupd}}$ region of the $\mathrm{Pt} / \mathrm{MFG}-\mathrm{H}$, showing a reliable depiction of the electrochemical surface phenomena on Pt. Thus, we can have shown that we can reliably study Pt/MFG-H by EQCN.

\section{Conclusions}

From these preliminary results, it is shown that the behavior of the carbon substrates is in good agreement with each other based on XRD diffraction patterns and XPS valence band structure data. The electrochemical surface response is different and was attributed to the orientation of the graphite crystal facets, as the ferri/ferrocyanide redox couple is very surface sensitive. However, the electrochemical response of the ferri/ ferrocyanide couple was in good agreement with the larger graphite crystallite size recorded by XRD. Furthermore, the $k^{0}$ and capacitance of the MFG, catalyzed and uncatalyzed, increases after surface modification. This is in accordance with the trend seen between modified and unmodified HOPG, and thus serves as a basis of comparison. Based on these findings, HOPG and MFG samples are structurally comparable, and their electrochemical behavior, while not identical, follows similar trends after modification.

In the case of Pt/MFG, the electrochemical surface response is less comparable to $\mathrm{Pt} / \mathrm{HOPG}$ when probing with the ferri/ ferrocyanide redox couple, but is similar in acidic media. It is 
postulated that the marginal physical surface differences in the MFG from HOPG are amplified by the deposition of Pt due to a more pronounced implantation of Pt into MFG than into HOPG. Work is ongoing to better understand why this difference occurs and to determine the influence of the Pt deposition and surface oxidation. More interestingly, Pt/MFG-H shows the expected frequency response curve shape, although further investigations are needed to better understand the repulsion and attractive forces that result in a higher physical frequency response.

Overall, the early results obtained for HOPG and MFG samples with $\mathrm{Pt}$ deposited are qualitatively comparable. With the EQCN analysis, we show that we have not changed the resonance of the quartz crystal and we can prepare samples using the MFG substrate as we do for HOPG. The EQCN technique may now be used to better study Pt nanoparticles supported on a carbon substrate and tentative inferences made to practical 3-D applications.

Acknowledgements AF would like to extend gratitude for support and training in the EQCN technique to Prof. Gregory Jerkiewicz and Dr. Jutae Kim at Queen's University, Department of Chemistry, 90 Bader Lane, Kingston, Ontario K7L 3N6, Canada. We thank the South African Department of Science and Technology for financial support in the form of HySA/Catalysis Centre of Competence Programme funding and a HySA/Catalysis student bursary (AF). This work is based on the research supported in part by the National Research Foundation (NRF) of South Africa (Grant Numbers 93205, EQP13091742446, and 92573). Authors further thank the NRF National Equipment Programme for access to equipment. Opinions expressed and conclusions arrived at are those of the authors and are not necessarily to be attributed to the NRF.

Open Access This article is distributed under the terms of the Creative Commons Attribution 4.0 International License (http:// creativecommons.org/licenses/by/4.0/), which permits unrestricted use, distribution, and reproduction in any medium, provided you give appropriate credit to the original author(s) and the source, provide a link to the Creative Commons license, and indicate if changes were made.

\section{References}

1. D.A. Buttry, M.D. Ward, Measurement of interfacial processes at electrode surfaces with the electrochemical quartz crystal microbalance. Chem. Rev. 92(6), 1355-1379 (1992)

2. W. Schmickler, Interfacial electrochemistry (Oxford University Press, Oxford, 1996)

3. Y. Ratieuville, P. Viers, J. Alexandre, G. Durand, A new electrochemical cell adapted to quartz crystal microbalance measurements. Electrochem. Commun. 2(12), 839-844 (2000)

4. G. Vatankhah, J. Lessard, G. Jerkiewicz, A. Zolfaghari, B.E. Conway, Dependence of the reliability of electrochemical quartzcrystal nanobalance mass responses on the calibration constant, $\mathrm{C}_{\mathrm{f}}$ : analysis of three procedures for its determination. Electrochim. Acta 48(11), 1613-1622 (2003)

5. C.A. Jeffrey, W.M. Storr, D.A. Harrington, Electrochemical quartzcrystal microbalance study of silver and copper electrodeposition on bare and iodine-covered platinum electrodes. J. Electroanal. Chem. 569(1), 61-70 (2004)
6. B. Keita, L. Nadjo, D. Belanger, C.P. Wilde, M. Hilaire, Electrochemical quartz crystal microbalance: evidence for the adsorption of heteropoly and isopoly anions on gold electrodes. J. Electroanal. Chem. 384(1), 155-169 (1995)

7. N. Gu, L. Niu, S. Dong, Simultaneous determination of both the calibration constant in an electrochemical quartz crystal microbalance and the active surface area of a polycrystalline gold electrode. Electrochem. Commun. 2(1), 48-50 (2000)

8. J. Janata, M. Josowicz, P. Vanýsek, D.M. DeVaney, Chemical sensors. Anal. Chem. 70(12), 179-208 (1998)

9. K. Bizet, C. Gabrielli, H. Perrot, Immunodetection by quartz crystal microbalance. Appl. Biochem. Biotechnol. 89(2), 139-149 (2000)

10. T. Wink, S.J. Van Zuilen, A. Bult, W.P. Van Bennekom, Selfassembled monolayers for biosensors. Analyst 122(4), 43R50R (1997)

11. G. Jerkiewicz, G. Vatankhah, J. Lessard, M.P. Soriaga, Y.-S. Park, Surface-oxide growth at platinum electrodes in aqueous $\mathrm{H}_{2} \mathrm{SO}_{4}$ : reexamination of its mechanism through combined cyclic-voltammetry, electrochemical quartz-crystal nanobalance, and Auger electron spectroscopy measurements. Electrochim. Acta 49(9-10), 1451-1459 (2004)

12. G. Jerkiewicz, Electrochemical hydrogen adsorption and absorption part 1: under-potential deposition of hydrogen. Electrocatalysis 1(4), 179-199 (2010)

13. G. Jerkiewicz, G. Vatankhah, S.-i. Tanaka, J. Lessard, Discovery of the potential of minimum mass for platinum electrodes. Langmuir 27(7), 4220-4226 (2011)

14. M. Watanabe, H. Uchida, in Electrocatalysis at platinum and bimetallic alloys, in fuel cell catalysis: a surface science approach, ed. by M. T. M. Koper. (Wiley, Hoboken, 2009), pp. 317-344

15. A.B. Ofstad, M.S. Thomassen, J.L. Gomez Dela Fuente, F. Seland, S. Møller-Holst, S. Sunde, Assessment of platinum dissolution from a Pt/C fuel cell catalyst: an electrochemical quartz crystal microbalance study. J. Electrochem. Soc. 157(5), B621-B627 (2010)

16. R.L. McCreery, K.K. Cline, C.A. McDermott, M.T. McDermott, Control of reactivity at carbon electrode surfaces. Colloids Surf. A Physicochem. Eng. Asp. 93(December), 211-219 (1994)

17. K.K. Cline, M.T. McDermott, R.L. McCreery, Anomalously slow electron transfer at ordered graphite electrodes: influence of electronic factors and reactive sites. J. Phys. Chem. 98(20), 5314-5319 (1994)

18. G. Jerkiewicz, G. Vatankhah, A. Zolfaghari, J. Lessard, Analysis of the mass response of the electrochemical quartz-crystal nanobalance in horizontal and vertical geometry. Electrochem. Commun. 1(9), 419-424 (1999)

19. U.J. Kim, C.A. Furtado, X. Liu, G. Chen, P.C. Eklund, Raman and IR spectroscopy of chemically processed single-walled carbon nanotubes. JACS 127(44), 15437-15445 (2005)

20. A. Falch, V. Lates, R.J. Kriek, Combinatorial plasma sputtering of $\mathrm{Pt}_{\mathrm{x}} \mathrm{Pd}_{\mathrm{y}}$ thin film electrocatalysts for aqueous $\mathrm{SO}_{2}$ electro-oxidation. Electrocatalysis 6(3), 322-330 (2015)

21. P. Scherrer, Bestimmung der größe und der inneren struktur von kolloidteilchen mittels Röntgenstrahlen. Nachr. Ges. Wiss. Gottingen, Math. Phys. 2(July), 98-100 (1918)

22. S. Evans, J.M. Thomas, The chemical nature of ion-bombarded carbon: a photoelectron spectroscopic study of "cleaned" surfaces of diamond and graphite. Proc. R. Soc. Lond. A Math. Phys. Sci. 353(1672), 103-120 (1977)

23. S.D. Gardner, C.S.K. Singamsetty, G.L. Booth, G.-R. He, C.U. Pittman, Surface characterization of carbon fibers using angleresolved XPS and ISS. Carbon 33(5), 587-595 (1995)

24. Y. Xie, P.M.A. Sherwood, X-ray photoelectron-spectroscopic studies of carbon fiber surfaces. 11. Differences in the surface chemistry and bulk structure of different carbon fibers based on poly (acrylonitrile) and pitch and comparison with various graphite samples. Chem. Mater. 2(3), 293-299 (1990) 
25. Z.R. Yue, W. Jiang, L. Wang, S.D. Gardner, C.U. Pittman Jr., Surface characterization of electrochemically oxidized carbon fibers. Carbon 37(11), 1785-1796 (1999)

26. A.J. Bard, L.R. Faulkner, Electrochemical methods: fundamentals and applications, 2nd edn. (John Wiley \& Sons, Inc., Hoboken, 2001)

27. R.S. Nicholson, Theory and application of cyclic voltammetry for measurement of electrode reaction kinetics. Anal. Chem. 37(11), 1351-1355 (1965)

28. K.J.J. Mayrhofer, B.B. Blizanac, M. Arenz, V.R. Stamenkovic, P.N. Ross, N.M. Markovic, The impact of geometric and surface electronic properties of Pt-catalysts on the particle size effect in electrocatalysis. J. Phys. Chem. B 109(30), 1443314440 (2005)

29. G. Jerkiewicz, M. DeBlois, Z. Radovic-Hrapovic, J.-P. Tessier, F. Perreault, J. Lessard, Underpotential deposition of hydrogen on benzene-modified $\mathrm{Pt}(111)$ in aqueous $\mathrm{H}_{2} \mathrm{SO}_{4}$. Langmuir 21(8), 3511-3520 (2005)

30. J. Kim, A. Munro, D. Beauchemin, G. Jerkiewicz, Limits of detection and quantification of electrochemical quartz-crystal nanobalance in platinum electrochemistry and electrocatalysis research. Anal. Chem. 88(21), 10599-10604 (2016) 